\title{
Model for the interpretation of nuclear magnetic resonance relaxometry of hydrated porous silicate materials
}

\author{
D. A. Faux, ${ }^{*}$ S.-H. P. Cachia, P. J. McDonald, J. S. Bhatt, and N. C. Howlett \\ Department of Physics, University of Surrey, Guildford GU2 7XH, United Kingdom \\ S. V. Churakov \\ Laboratory of Waste Management, Paul Scherrer Institute, CH-5232 Villigen, Switzerland
}

(Received 21 November 2014; published 20 March 2015)

\begin{abstract}
Nuclear magnetic resonance (NMR) relaxation experimentation is an effective technique for probing the dynamics of proton spins in porous media, but interpretation requires the application of appropriate spindiffusion models. Molecular dynamics (MD) simulations of porous silicate-based systems containing a quasitwo-dimensional water-filled pore are presented. The MD simulations suggest that the residency time of the water on the pore surface is in the range $0.03-12 \mathrm{~ns}$, typically $2-5$ orders of magnitude less than values determined from fits to experimental NMR measurements using the established surface-layer (SL) diffusion models of Korb and co-workers [Phys. Rev. E 56, 1934 (1997)]. Instead, MD identifies four distinct water layers in a tobermorite-based pore containing surface $\mathrm{Ca}^{2+}$ ions. Three highly structured water layers exist within $1 \mathrm{~nm}$ of the surface and the central region of the pore contains a homogeneous region of bulklike water. These regions are referred to as layer 1 and 2 (L1, L2), transition layer (TL), and bulk (B), respectively. Guided by the MD simulations, a two-layer (2L) spin-diffusion NMR relaxation model is proposed comprising two two-dimensional layers of slow- and fast-moving water associated with $\mathrm{L} 2$ and layers $\mathrm{TL}+\mathrm{B}$, respectively. The $2 \mathrm{~L}$ model provides an improved fit to NMR relaxation times obtained from cementitious material compared to the SL model, yields diffusion correlation times in the range 18-75 ns and 28-40 ps in good agreement with MD, and resolves the surface residency time discrepancy. The $2 \mathrm{~L}$ model, coupled with NMR relaxation experimentation, provides a simple yet powerful method of characterizing the dynamical properties of proton-bearing porous silicate-based systems such as porous glasses, cementitious materials, and oil-bearing rocks.
\end{abstract}

DOI: 10.1103/PhysRevE.91.032311

\section{INTRODUCTION}

Proton nuclear magnetic resonance (NMR) relaxation analysis is the most powerful technique for probing the dynamics of hydrogen-bearing molecules in porous media with applications in disciplines as diverse as petroleum engineering, catalysis, polymer science, biology, glass science, and construction materials. NMR relaxation analysis is able to provide information on the motion of hydrogen $\left({ }^{1} \mathrm{H}\right)$ protons in porous materials at the nanoscale, to infer pore-size distributions, pore surface-to-volume ratios, the time scale for pore filling and emptying, and to infer the diffusion mechanism for the mobile molecules.

According to the Bloembergen, Purcell, and Pound (BPP) model [1], the NMR spin-lattice and spin-spin relaxation times, $T_{1}$ and $T_{2}$, depend on fluctuations in magnetic dipole-dipole interactions between proton spins (usually labeled as $I$ spins) on the same molecule (intramolecular or rotational), different molecules (intermolecular translations), and between mobile $I$ spins and static paramagnetic impurities, labeled as $S$ spins. Thus the interpretation of NMR relaxation experiments depends on models describing the movement of the protonbearing fluid. Time-dependent dipole-dipole correlation functions $G(t)$ derived from a model are Fourier transformed to obtain the NMR $T_{1}$ and $T_{2}$ relaxation dispersions for comparison with experiment. One family of widely used models has been pioneered by Korb and co-workers over

*Corresponding author: d.faux@surrey.ac.uk
PACS number(s): 82.33.Ln, 76.60.-k, 68.08.-p, 47.11.Mn the last 15-20 years [2-9]. An early work [3] provided a simplified model for the relaxation associated with the $I-S$ spin interactions between diffusing protons contained in a quasi-two-dimensional (Q2D) volume of fluid and a dilute concentration of static paramagnetic impurities on the pore surface, chiefly $\mathrm{Fe}^{3+} . \mathrm{Fe}^{3+}$ ions are present in many porous silicate materials by the substitution of silicon atoms and, although concentrations are low, the $I-S$ spin interactions dominate the dipolar relaxation at low magnetic field strengths. Therefore, models of the relaxation in cementitious materials, rocks, many glasses, and silica-based granular material include, as the primary relaxation mechanism, the interaction of the mobile $I$ spins with the static $S$ impurities.

The models of Korb and co-workers isolate the separate contributions to the relaxation of spins diffusing in the bulk volume of the pore and spins adsorbed to the pore surface. Assuming exchange between the pore surface and the pore bulk that is faster than the $T_{1}^{-1}$ and $T_{2}^{-1}$ relaxation rates (the biphasic fast-exchange regime), the experimental relaxation rates $T_{1}^{-1}$ and $T_{2}^{-1}$ are the average of that for the surface fluid and the bulk fluid weighted by the number of spins contributing to the relaxation in each environment [10]. In 2001, Godefroy et al. [4] argued that the dominant contribution to the dipolar relaxation in silica carbide granular media was associated solely with the interaction between adsorbed mobile $I$ spins and the electronic spin associated with rare $\mathrm{Fe}^{3+}$ paramagnetic impurities located on the surface of the pore. The surface $I-S$ interaction was shown to dominate due to a $t^{-1}$ dependence of the dipolar correlation function $G(t)$ at long times compared to a $t^{-2}$ dependence due to the dipolar interaction between bulk 


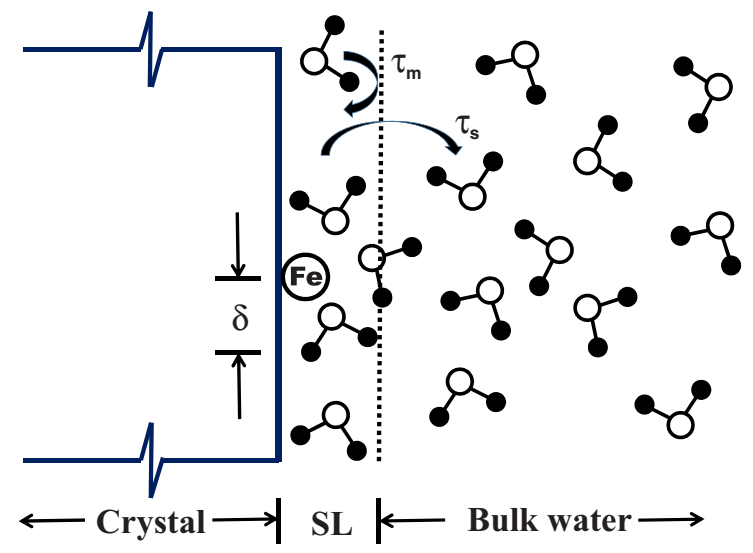

FIG. 1. (Color online) A schematic representation of the surface layer (SL) model presented by Korb and co-workers [3,4]. Water diffuses with a correlation time $\tau_{m}$ in the surface layer of a pore containing a dilute concentration of $\mathrm{Fe}^{3+}$ paramagnetic impurities. Water desorbs into the pore bulk with a characteristic time $\tau_{s}$ and ceases to contribute to the dipolar relaxation thereafter. $\delta \approx 0.27 \mathrm{~nm}$ is the approximate distance of closest approach of a water proton to a $\mathrm{Fe}^{3+}$ impurity.

$I$ spins and surface $S$ spins. We refer to this model, illustrated in Fig. 1, as the surface-layer (SL) model.

According to the SL model, adsorbed proton-bearing molecules undergo a two-dimensional (2D) walk across the pore surface, making repeated encounters with surface paramagnetic impurities. The $I-S$ dipolar interaction is modulated by the translational motion of spin $I$ on the surface. The model invokes two correlation times: $\tau_{m}$, the correlation time for surface hops associated with the 2D walk of the $I$ spins on the surface, and $\tau_{s}$, the surface residency time which is a measure of surface affinity. Experiments were undertaken by Godefroy et al. [4] on hydrated granular porous systems comprised of micron-sized grains of $\mathrm{SiC}$. The pore surfaces were composed of silica $\left(\mathrm{SiO}_{2}\right), \tau_{m}$ was obtained by fitting the SL model to the frequency dependence of $T_{1}^{-1}$ and found to be $0.6 \mathrm{~ns} . \tau_{s}$ was estimated from the high-frequency limit of $T_{1}^{-1}$ as $1.6 \mu \mathrm{s}$. Later, the SL model was successfully fitted to $T_{1}^{-1}$ over three orders of magnitude of frequency $(20 \mathrm{kHz}$ to $20 \mathrm{MHz}$ ) for a mortar [5] and to the ratio $T_{2} / T_{1}$ for white cement paste [6]. The frequency dependence of $T_{1}^{-1}$ is particularly sensitive to $\tau_{m}$ and consistent values for $\tau_{m}$ are obtained for a range of porous materials with silica-based pore surfaces. For example, in the case of calcium silicate hydrates $(\mathrm{C}-\mathrm{S}-\mathrm{H})$, the active phase in cement materials [11,12], $\tau_{m}$ is of the order of 1.3 ns [5]. The relaxation times per se are much less sensitive to $\tau_{s}$, but it was nevertheless established that $\tau_{s} \gg \tau_{m}$ [4-6]. However, the ratio of $T_{2} / T_{1}$ depends critically on $\tau_{s}$. A factor of about 0.25 at $20 \mathrm{MHz}$ is typical for C-S-H and leads to an estimate of $\tau_{s}=13 \mu \mathrm{s}$ [6], which is close to the value for the hydrated $\mathrm{SiC}$ granular system of Godefroy et al. [4].

Despite the success of the SL model in interpreting NMR relaxation data and in yielding consistent values of $\tau_{m}$ and $\tau_{s}$ for a variety of systems, the validity of the model has been questioned. The estimate of $\tau_{s}=13 \mu$ s for the surface residency time in cementitious material is long and indicates that a water molecule makes approximately 10000 hops before desorbing. It is a challenge to devise a mechanism that allows water molecules to be sufficiently mobile to traverse distances of typically $30 \mathrm{~nm}$, yet to be bound to the surface throughout. Moreover, the SL model assumes that spins which desorb to the pore bulk do not contribute to the relaxation thereafter. In other words, it is assumed that a desorbed spin does not return to the surface on a time scale much longer than the surface residency time of $13 \mu \mathrm{s}$. Yet, a bulk water molecule diffuses a distance of about $0.4 \mu \mathrm{m}$ in $13 \mu \mathrm{s}$, a distance which is much larger than the typical pore thickness in cementitious materials where, despite uncertainty as to the morphology of mature $\mathrm{C}-\mathrm{S}-\mathrm{H}$, most pores have sizes ranging from about 2 to $50 \mathrm{~nm}[7,13,14]$. Furthermore, the fast-exchange model has limited application if $\tau_{s} \approx 10 \mu \mathrm{s}$. For a pore of thickness equivalent to 10 surface layers, for example, mixing of surface and bulk water can only occur over time scales longer than $\approx 10 \times 10 \mu \mathrm{s}$, which is similar to typical spin-spin relaxation times $T_{2}$ of ${ }^{1} \mathrm{H}$ in C-S-H, i.e., about $100 \mu$ s [14].

This work is motivated by the desire to resolve the above inconsistencies, to identify the nanoscale structure of $\mathrm{C}-\mathrm{S}-\mathrm{H}$ in cement, and, in particular, to understand the behavior of water in the nanoscale pores formed by C-S-H. This is vital if cementitious materials are to be developed with improved durability and lower carbon footprint, bearing in mind that cement production worldwide currently contributes $5-8 \%$ of global $\mathrm{CO}_{2}$ emissions and is set to rise further as demand increases in the developing nations. The detailed morphology of C-S-H is still debated but it is widely acknowledged that C$\mathrm{S}-\mathrm{H}$ is a highly heterogeneous, nanoporous material comprised of disordered sheets of calcium and oxygen atoms and silicate tetrahedra separated by sheets of water further separated by nanosized pores, i.e., so-called gel pores, and larger capillary pores $[13,15]$. Although the focus of the present work is on cementitious materials, many of the conclusions are equally applicable to other nanoporous silicate-based materials such as zeolites, glasses, and quartz.

In this paper, the results of molecular dynamics (MD) simulations of hydrated cement-analogue Q2D pores are presented. Previous MD simulations of cement-analogue mineral-water interfaces have been undertaken by Kirkpatrick and co-workers [16], Pellenq and co-workers [17], Nonat and co-workers [18], and others. A good review may be found in Ref. [19]. Of particular interest is the comparison of the surface diffusion coefficient between the MD simulation and NMR experimentation [20] characterized in the SL model by a surface diffusion correlation time $\tau_{m}$ using a modified tobermorite $9 \AA$ cement analog. Tobermorite is a silicatebased material with a well-characterized structure used to model stacked C-S-H layers. The surface diffusion correlation time $\tau_{m}$ was found to be in the range 0.1-1.1 ns (with an average of $0.2 \mathrm{~ns}$ ), which is consistent with experiment and serves to validate both the experimental results and the MD approach.

We present results of MD simulations of water in Q2D pores confined by a silica or tobermorite crystal with a focus on the measurement of the surface residency time, $\tau_{s}$. A variety of MD model systems are explored, but in all cases $\tau_{s}$ is found to be shorter than the value obtained by fitting the SL model to NMR results by $2-5$ orders of magnitude. Furthermore, the MD simulations do not reveal large-scale surface diffusion of 
water prior to desorption. Both observations conflict with the SL model.

In one MD simulation, the water is confined to a $14 \AA$ tobermorite crystal with surfaces modified by replacing bridging $\mathrm{SiO}_{2}$ tetrahedra by calcium ions. The $\mathrm{Ca} / \mathrm{Si}$ ratio is therefore 1.5 , which is close to that observed in experiment. This simulation reveals three highly structured water layers and a homogeneous region of bulklike water in the central region of the pore. Layer 1 (L1) contains adsorbed water located between the crystal surface and a layer of aqueous $\mathrm{Ca}^{2+}$ ions. The water in layer 2 (L2) forms the first hydration shell to the adsorbed $\mathrm{Ca}^{2+}$. Layer 3 is formed by water molecules in the second hydration shell of $\mathrm{Ca}^{2+}$ ions referred to as the transition layer (TL).

A simplified two-layer model is presented in which the motion of the water is described by two representative 2D layers of mobile water. We refer to this model as the $2 \mathrm{~L}$ model. The theory is developed resulting in a nearly analytic expression for the dipolar correlation function representing the interaction between mobile $I$ spins contained in a 2D layer displaced from the static relaxation centers close to the crystal surface. It is demonstrated that the $2 \mathrm{~L}$ model not only provides an improved fit to the experimental data presented by Barberon and co-workers [5] and McDonald and co-workers [6], but also yields estimates of physical quantities that are consistent with simulation.

The structure of the paper is as follows. The SL model is reviewed in Sec. II B. The theory for the spin-lattice and spin-spin relaxation times $T_{1}$ and $T_{2}$ associated with 2D translational diffusion of $I$ spins in a plane displaced from the paramagnetic relaxation centers is presented in Sec. II C. Section III summarizes the key results from the MD simulations. Previous experimental results are then reanalyzed using the 2L model in Sec. IV and, finally, the key results are summarized in Sec. V.

\section{THEORY}

\section{A. General background}

A collection of $I$ spins diffuse in a pore space in the presence of a static magnetic field. Nuclear spin relaxation arises due to the modulation of the dipolar interaction between the $I$ spins and fixed paramagnetic impurities ( $S$ spins) due to the relative translational diffusion and rotational motion of spin $I$ relative to spin $S$. The spin-lattice and spin-spin relaxation times associated with the translational motion of spin $I$ with respect to spin $S$, i.e., $T_{1, I S}$ and $T_{2, I S}$, respectively, may be expressed in terms of the powder-average spectral density function $J(\omega)$ as [6,21]

$$
\begin{gathered}
T_{1, I S}^{-1}=\frac{1}{3} \beta_{I S}^{2}\left[7 J_{I S}\left(\omega_{S}\right)+3 J_{I S}\left(\omega_{I}\right)\right], \\
T_{2, I S}^{-1}=\frac{1}{6} \beta_{I S}^{2}\left[4 J_{I S}(0)+13 J_{I S}\left(\omega_{S}\right)+3 J_{I S}\left(\omega_{I}\right)\right],
\end{gathered}
$$

where $\beta_{I S}=\left(\mu_{0} / 4 \pi\right) \gamma_{I} \gamma_{S} \hbar \sqrt{S(S+1)}$, and $\gamma_{I}$ and $\gamma_{S}$ are the gyromagnetic ratios for spin $I$ and $S$, respectively, with $S=\frac{5}{2}$ for $\mathrm{Fe}^{3+}$. The Larmor frequency of spin $I$ in the applied static field is $\omega_{I}$ and $\omega_{S}=658.21 \omega_{I}$. The ratio of the relaxation times is therefore

$$
\frac{T_{2, I S}}{T_{1, I S}}=\frac{2\left[7 J_{I S}\left(\omega_{S}\right)+3 J_{I S}\left(\omega_{I}\right)\right]}{4 J_{I S}(0)+13 J_{I S}\left(\omega_{S}\right)+3 J_{I S}\left(\omega_{I}\right)},
$$

which is Eq. (5) presented by McDonald et al. [6]. The powder-average spectral density function $J_{I S}(\omega)$ is obtained from the Fourier transformation of the powder-average dipolar correlation function $G_{I S}(t)$ defined as

$$
J_{I S}(\omega)=2 \int_{0}^{\infty} G_{I S}(t) \cos \omega t d t .
$$

It is therefore necessary to determine the dipolar correlation function $G_{I S}(t)$ for the translational diffusion of $I$ spins for a chosen diffusion model.

A mobile spin confined to a pore may be located in one of two distinct environments, the surface or bulk. The observed relaxation rate may be expressed as

$$
T_{1, \mathrm{obs}}^{-1}=x T_{1, \mathrm{surf}}^{-1}+(1-x) T_{1, \mathrm{bulk}}^{-1},
$$

where $x=N_{\text {surf }} / N$, and $N=N_{\text {surf }}+N_{\text {bulk }}$ is the fraction of the total number of spins in the surface region. There is an equivalent expression for $T_{2 \text {,obs }}^{-1}$.

\section{B. The surface-layer (SL) model}

The SL model introduced by Korb and co-workers [3,4] is presented schematically in Fig. 1 and is applicable to systems in which the dominant relaxation mechanism is associated with the interaction between a uniform density of static $S$ spins and a uniform density of $I$ spins undertaking uncorrelated translational motion in the $2 \mathrm{D}$ surface of the pore. In the SL model, both the $I$ spins and the $S$ spins are confined to the same 2 D surface plane. The distance of nearest approach of spin $I$ to spin $S$ in the 2D plane is defined as $\delta$, which is typically in the range $0.27-0.30 \mathrm{~nm}$ [5]. $\delta$ acts as a convenient unit of elementary distance in the SL model, but the primary results from the model do not depend on the choice of $\delta$.

The SL model neglects all $I-I$ spin interactions for surface-surface interactions on the basis that $\gamma_{\mathrm{S}} \gg \gamma_{\mathrm{I}}$. All $I-S$ interactions for subsurface $S$ spins in the crystal are neglected. Contributions to observed relaxation rates due to the bulk water confined in the pores are not explicitly included in the SL model, but this contribution is included as a single frequency-independent contribution when executing a fit to relaxation time data at low magnetic field [5].

Korb and co-workers showed that the translational motion of $I$ spins confined to a 2D layer leads to a $t^{-1}$ dependence of the dipolar correlation function $G_{I S, \mathrm{SL}}(t)$ at long times [3,4]. Consequently, the leading term for $G_{I S, \mathrm{SL}}(t)$ at long times is of the form $A t^{-1}$, where $A$ is a constant which is proportional to $\sigma_{S} \epsilon^{-4}$, where $\sigma_{S}$ is the areal density of $S$ spins at the 2D pore surface and $\epsilon$ is a distance parameter of order $\delta$. It is not possible to Fourier transform a function of the form $A t^{-1}$. Therefore, the SL model also assumes that the $I$ spins desorb from the surface (and cease to contribute to the relaxation) with a characteristic time $\tau_{s}$. After time $\tau_{s}$, the number of $I$ spins at the surface has dropped by a factor $e^{-1}$ compared to the number at the surface at $t=0$. On this basis, the following expression for $G_{I S, \mathrm{SL}}(t)$ was proposed [4]:

$$
G_{I S, \mathrm{SL}}(t)=\frac{A}{t}\left[\frac{e^{-t / \tau_{s}}-e^{-t / \tau_{m}}}{\tau_{m}^{-1}-\tau_{s}^{-1}}\right],
$$

where the correlation time $\tau_{m}$ is the characteristic time for a move of length $\delta$ of the $I$ spins within the 2D surface layer such 


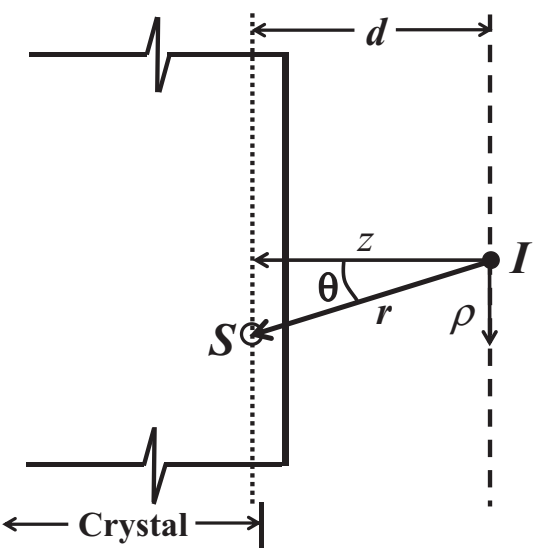

FIG. 2. A schematic diagram of a 2D layer containing diffusing $I$ spins which interact with static paramagnetic impurities ( $S$ spins) of areal density $\sigma_{S}$. The separation of the two layers is $d$.

that the self-diffusion coefficient $D=\delta^{2} / 4 \tau_{m}$. The expression for $G_{I S, \mathrm{SL}}(t)$ is dependent on three parameters, i.e., $A, \tau_{m}$, and $\tau_{s}$. Note that $A$ in Eq. (6) incorporates powder averaging and therefore differs from the expression in Ref. [4] by a factor 1/5. Equation (6) may be Fourier transformed analytically to yield $J_{I S, \mathrm{SL}}(\omega)$ and hence the NMR relaxation times may be calculated using Eqs. (1) and (2).

\section{C. $G(t)$ for a displaced 2D layer of diffusing spins}

We now determine the powder-average dipolar correlation function $G(t)$ for the translational motion of $I$ spins in a $2 \mathrm{D}$ plane placed parallel to a layer of $S$ spins separated by a perpendicular distance $d$. A schematic diagram is presented as Fig. 2. The system is identical to the SL model illustrated in Fig. 1, except that the layer containing the diffusing spins is displaced from the layer containing the $S$ spins. The starting point for the derivation of the correlation function $G(t)$ is the expression [22]

$$
\begin{aligned}
G(t)= & \frac{4 \pi N_{\mathrm{Fe}}}{5} \iint\left[\sum_{M=-2}^{2} \frac{Y_{2 M}\left(\theta_{0}, \phi_{0}\right) Y_{2 M}^{*}(\theta, \phi)}{r_{0}^{3} r^{3}}\right] \\
& \times P\left(\mathbf{r}, t \mid \mathbf{r}_{0}\right) d^{3} \mathbf{r}_{0} d^{3} \mathbf{r},
\end{aligned}
$$

where $P\left(\mathbf{r}, t \mid \mathbf{r}_{0}\right)$ is the probability density function (or diffusion propagator) for a pair of spins separated by $\mathbf{r}$ at time $t$ given the spin pair was separated by $\mathbf{r}_{0}$ at $t=0 . N_{\mathrm{Fe}}$ is the volume density of $S$ spins at the surface of the crystal, usually expressed as $N_{\mathrm{Fe}}=\sigma_{S} / \delta_{\mathrm{Fe}}$, where $\sigma_{s}$ is the areal density of $S$ spins contained in a thickness $\delta_{\mathrm{Fe}}$ of crystal. The $Y$ are the spherical harmonic functions of order two and the presence of the asterisk superscript on the $Y$ indicates the complex conjugate. The powder average has been taken resulting in the factor $1 / 5$ [22].

The probability density function appropriate for the diffusion of $I$ spins relative to fixed $S$ spins where the $I$ spins are confined to a layer of thickness $\delta$ may be written

$$
P\left(\mathbf{r}, t \mid \mathbf{r}_{0}\right)=\frac{1}{\delta} \frac{1}{4 \pi D t} e^{-\left|\boldsymbol{\rho}-\boldsymbol{\rho}_{0}\right|^{2} / 4 D t},
$$

where the appearance of $1 / \delta$ arises due to the assumption of a uniform density of $I$ spins across the layer thickness $\delta$, where $\delta$ is taken to be the approximate distance of nearest approach of protons on different water molecules. The $\boldsymbol{\rho}$ represents a vector in the plane of the layer. $D$ is the $2 \mathrm{D}$ self-diffusion coefficient of the $I$ spins, which may be expressed in terms of a characteristic diffusion correlation time $\tau$ by

$$
D=\frac{\delta^{2}}{4 \tau} \text {. }
$$

Thus, $\tau$ is the mean time for a move of distance $\delta$ in the 2D plane. Note that this definition is slightly different from that for the SL model. In the SL model, $\delta$ is the $I-S$ distance of nearest approach [5], although the numerical value is the same in each model, namely, $0.27 \mathrm{~nm}$.

Equation (8) is replaced by its well-known Fourier integral with Fourier variable $\mathbf{k}$ and then substituted into Eq. (7) to yield

$$
\begin{aligned}
G(t)= & \frac{4 \sigma_{S} \delta}{5 \pi \delta_{\mathrm{Fe}}} \iint \sum_{M=-2}^{2} \frac{Y_{2 M}\left(\theta_{0}, \phi_{0}\right) Y_{2 M}^{*}(\theta, \phi)}{\left(\rho_{0}^{2}+d^{2}\right)^{3 / 2}\left(\rho^{2}+d^{2}\right)^{3 / 2}} \\
& \times\left[\int e^{-D t k^{2}} e^{i \mathbf{k} \cdot \boldsymbol{\rho}} e^{-i \mathbf{k} \cdot \boldsymbol{\rho}_{0}} d^{2} \mathbf{k}\right] d^{2} \boldsymbol{\rho}_{0} d^{2} \boldsymbol{\rho},
\end{aligned}
$$

where $r^{2}=\rho^{2}+d^{2}, d^{2} \boldsymbol{\rho} \equiv \rho d \rho d \phi$ and the integrations over $z$ and $z_{0}$ have been completed. The exponential functions are replaced using the Jacobi-Anger expression and the functional forms for the spherical harmonic functions inserted. Integrals involving $M= \pm 1$ vanish and the integration over the azimuthal angles $\phi$ and $\phi_{0}$ may be undertaken explicitly. Simplifications lead to the following expression:

$$
G(t)=\frac{\pi \sigma_{S} \delta}{4 \delta_{\mathrm{Fe}}} \int_{0}^{\infty} k e^{-D t k^{2}}\left[6 I_{2}^{2}(k)+2 I_{0}^{2}(k)\right] d k,
$$

where

$$
\begin{aligned}
& I_{0}(k)=\int_{0}^{\infty} \frac{\rho\left(2 d^{2}-\rho^{2}\right)}{\left(\rho^{2}+d^{2}\right)^{3 / 2}} J_{0}(k \rho) d \rho, \\
& I_{2}(k)=\int_{0}^{\infty} \frac{\rho^{3}}{\left(\rho^{2}+d^{2}\right)^{3 / 2}} J_{2}(k \rho) d \rho,
\end{aligned}
$$

and where the subscripts on $I_{0}$ and $I_{2}$ reflect their origin as $M=$ 0 and $M=2$ terms, respectively. Here, $J_{0}$ and $J_{2}$ are Bessel functions of the first kind. Changing variables to dimensionless quantities based on a distance $\epsilon$, such that $\kappa=k \epsilon, p=\rho / \epsilon$, and $\xi=d / \epsilon$, and executing the integrations with the aid of Gradsteyn and Ryzhik [23], gives

$$
I_{0}(k)=\frac{\kappa e^{-\xi \kappa}}{\epsilon}, \quad I_{2}(k)=\frac{\kappa e^{-\xi \kappa}}{3 \epsilon} .
$$

Substituting these expressions into Eq. (11) and use of Eq. (9) yields the final expression for $G(t)$,

$$
\begin{aligned}
G(t) & =\frac{2 \pi \sigma_{S} \delta}{3 \epsilon^{4} \delta_{\mathrm{Fe}}} K(t, \tau), \\
K(t, \tau) & =\int_{0}^{\infty} \kappa^{3} e^{-\kappa^{2} t / 4 \tau} e^{-2 \xi \kappa} d \kappa .
\end{aligned}
$$

The behavior of the correlation function at long times may be found by setting the upper limit on the integration to $\Delta$ and 
making the substitution $v=\kappa \sqrt{t}$ to yield

$$
K(t \rightarrow \infty, \tau)=\frac{1}{t^{2}} \int_{0}^{\Delta \sqrt{t}} v^{3} e^{-v^{2} / 4 \tau} e^{-2 \xi v / \sqrt{t}} d v .
$$

Executing the integral and setting $t \rightarrow \infty$ produces a leading term for $G(t \rightarrow \infty)$ of the form

$$
G(t \rightarrow \infty)=\frac{16 \pi \sigma_{S} \delta \tau^{2}}{3 \epsilon^{4} \delta_{\mathrm{Fe}} t^{2}},
$$

which is independent of both $\Delta$ and $\xi$. Equation (16) is fundamentally different from the equivalent expression for the SL model as given by Eq. (6) due to the displacement of the 2D layer containing $I$ spins from the layer containing the relaxation centers. If all $I-S$ spin vectors are contained in a plane, $\theta=\pi / 2$, and the spherical harmonic functions are simplified, resulting in a leading term proportional to $t^{-1}$. This simplification is not valid when the diffusing spins are displaced from the $S$ spins and the leading term at long times becomes proportional to $t^{-2}$. This $t^{-2}$ dependence was effectively obtained by Korb and co-workers in their analysis of $I-S$ spin relaxation in Q2D pores [3].

\section{RESULTS OF MOLECULAR DYNAMICS SIMULATION}

The aim of the present MD simulations is to elucidate the atomic-scale dynamics of water on cement-analogue surfaces, thereby testing the validity of the assumptions of the SL models. MD simulations of hydrated silicate-based pores were undertaken exploring a range of crystals, surface morphologies, pore sizes, and potential energy functions, as summarized in Table I. The five simulations are labeled MD1-MD5. The confining crystals were silica, tobermorite $14 \AA$, or tobermorite $11 \AA$ [24-26]. Tobermorite has a well-characterized crystal structure and is frequently used as an analog for stacked C-S-H layers [19]. Water was confined by the crystal to a Q2D pore with thicknesses ranging from 1.0 to $5.0 \mathrm{~nm}$. As an example, model MD4 is illustrated in Fig. 3. The MD simulations were performed using the DL_POLY package $[27,28]$ with periodic

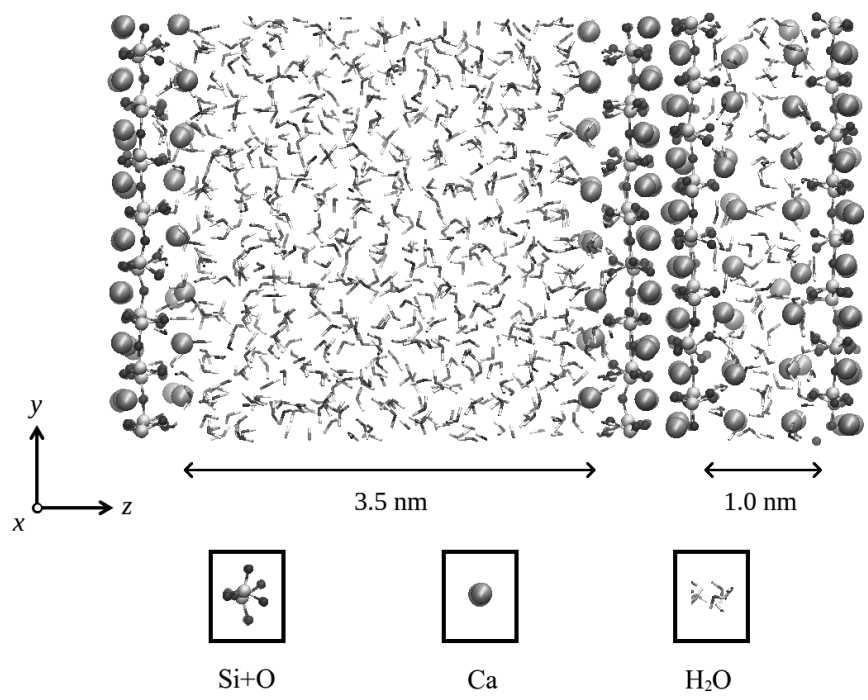

FIG. 3. A simplified image taken from molecular dynamics model MD4 showing a pore with water confined in the $z$ direction by modified tobermorite $14 \AA$ surfaces. The Si atoms are fixed, the surface oxygen atoms are tethered, and $\mathrm{Ca}^{2+}$ ions are present near the surfaces and are mobile.

boundary conditions applied in all three directions. Water molecules in the pore thus experience confinement in the $z$ direction, while the $x-y$ plane is infinite due to the periodic boundary conditions. The SPC/E [29] or TIP4P [30] potential was used for the water-water interactions and the CLAYFF [31] or modified Freeman [32-35] potential was applied for interactions within the crystal. The Lorentz-Berthelot mixing rules [36] were applied to model the crystal-water interface. Some details of models MD1 and MD2 have been published elsewhere [22,37].

Each system was equilibrated using the isothermal-isobaric ensemble to a target temperature of $300 \mathrm{~K}$ and a pressure of $0 \mathrm{~Pa}$. Water molecules were added or removed from the pore to match the bulk water density in the middle of the

TABLE I. List of molecular dynamics simulations with surface conditions, interatomic potentials, and results for the surface residency time

\begin{tabular}{|c|c|c|c|}
\hline Model & Description & Potentials & $\tau_{s}(\mathrm{~ns})$ \\
\hline MD1 & $\begin{array}{l}\text { alpha-quartz }\left(\mathrm{SiO}_{2}\right) \text { with } \mathrm{Si}, \mathrm{O} \text { mobile } \\
\text { (100) surface terminated with O } \\
\text { Pore } 1.0 \mathrm{~nm} \text { containing } 886 \mathrm{H}_{2} \mathrm{O}\end{array}$ & $\begin{array}{l}\text { CLAYFF } \\
\text { SPC/E }\end{array}$ & 0.32 \\
\hline MD2 & $\begin{array}{l}\text { alpha-quartz } \mathrm{SiO}_{2} \text { with } \mathrm{Si}, \mathrm{O} \text { mobile. } \\
\text { (100) surface with surface hydroxyls (H tethered) } \\
\text { Pore } 1.0 \mathrm{~nm} \text { containing } 886 \mathrm{H}_{2} \mathrm{O}\end{array}$ & $\begin{array}{l}\text { CLAYFF } \\
\text { SPC/E }\end{array}$ & 0.15 \\
\hline MD3 & $\begin{array}{l}11 \AA \text { anomalous tobermorite with } \mathrm{Si} \text {, O mobile } \\
\text { (100) surface with surface hydroxyls (H tethered) } \\
\text { Pore } 1.0 \mathrm{~nm} \text { containing } 792 \mathrm{H}_{2} \mathrm{O}\end{array}$ & $\begin{array}{l}\text { CLAYFF } \\
\text { SPC/E }\end{array}$ & 0.03 \\
\hline MD4 & $\begin{array}{l}14 \AA \text { tobermorite, } \mathrm{Si} / \mathrm{O} \text { frozen, bridging tetrahedra replaced by } \mathrm{Ca}^{2+} \\
\text { (100) surface terminated with } \mathrm{O} \text { (surface } \mathrm{O} \text { tethered) } \\
\text { Pore } 3.3 \mathrm{~nm} \text { containing } 745 \mathrm{H}_{2} \mathrm{O}, \mathrm{Ca} / \mathrm{Si}=1.5\end{array}$ & $\begin{array}{l}\text { CLAYFF } \\
\text { SPC/E }\end{array}$ & 12 \\
\hline MD5 & $\begin{array}{l}14 \AA \text { tobermorite, } \mathrm{Si} / \mathrm{O} \text { frozen, bridging tetrahedra replaced by } \mathrm{Ca}^{2+} \\
\text { (100) surface with surface hydroxyls }(\mathrm{H} \text { tethered) } \\
\text { Pore } 3.3 \mathrm{~nm} \text { containing } 745 \mathrm{H}_{2} \mathrm{O}, \mathrm{Ca} / \mathrm{Si}=1.5\end{array}$ & $\begin{array}{l}\text { modified } \\
\text { Freeman } \\
\text { TIP4P }\end{array}$ & 0.08 \\
\hline
\end{tabular}
$\tau_{s}$. Some details of models MD1 and MD2 have been published [22,37]. 
pore at equilibrium. A cutoff distance of $10.0 \AA$ was applied to the Lennard-Jones potentials and the Ewald summation method [38] was used to incorporate long-range Coulombic interactions. Each of these systems was then simulated under the NVT conditions (constant number of particles, volume, and temperature) using the Hoover thermostat [39] to obtain a MD trajectory up to $20 \mathrm{~ns}$ long.

A full analysis of the MD results will be reported elsewhere. Here we focus on the results for the physical quantities relevant to the models presented in Sec. II C and, for this purpose, we focus on model MD4 as the model C-S-H structure most suitable for later comparison with NMR relaxation results. However, our broad conclusions are not model (MD1-MD5) dependent. MD4 comprises a tobermorite $14 \AA$ surface in which the bridging tetrahedra have been replaced by $\mathrm{Ca}^{2+}$ ions. The calcium ions yield a $\mathrm{Ca} / \mathrm{Si}$ ratio of 1.5 , which is close to the experimental value of about 1.8 typical of cementitious materials. The silicon atoms in the crystal are frozen, but the surface oxygen atoms are mobile and tethered.

The surface of $\mathrm{C}-\mathrm{S}-\mathrm{H}$ carries a positive permanent charge due to the deprotonation of Si-OH surface sites. This surface charge causes structuring of water and adsorption of aqueous $\mathrm{Ca}^{2+}$ ions at the C-S-H surface [40]. The density distribution of water and aqueous $\mathrm{Ca}^{2+}$ ions in a $3.3 \mathrm{~nm}$ pore for the model MD4 is shown in Fig. 4. Three highly structured water layers within $1 \mathrm{~nm}$ to the surface and a homogeneous region of bulklike water can be clearly distinguished. These regions are referred to as layer 1 and 2 (L1, L2), transition layer (TL), and bulk (B), respectively. Analysis of current classical MD simulations and our former results from $a b$ initio modeling of C-S-H [40] and tobermorite [25,26] suggest that L1 is formed by $\mathrm{H}_{2} \mathrm{O}$ molecules adsorbed on the crystalline surface of $\mathrm{C}-\mathrm{S}-\mathrm{H}$ and water coordinating adsorbed aqueous $\mathrm{Ca}^{2+}$ ions. The L2 layer is formed by water in the first hydration shell of adsorbed $\mathrm{Ca}^{2+}$ ions. The maximum of the aqueous $\mathrm{Ca}^{2+}$ density profile is on the L1/L2 boundary. The TL is dominated

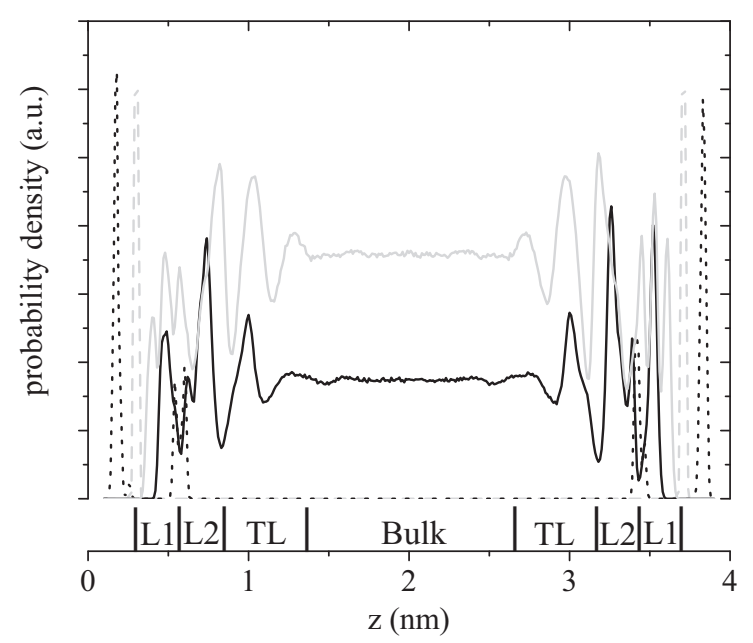

FIG. 4. The particle density is presented as a function of $z$ for the surface silicon atoms (gray dashed line), calcium in the crystal and pore (black dashed line), and the water-oxygen (black solid line) and the water-hydrogen (gray solid line) atoms in the pore. The water exhibits four layers labeled L1 (layer 1), L2 (layer 2), transition layer (TL), and bulk (B). by water molecules forming the second hydration shell of the adsorbed $\mathrm{Ca}^{2+}$ ions. The density profile of water oxygen next to the surface is consistent with that obtained by Cheng and co-workers from a hydrated mica surface using x-ray reflectivity [41] and in accord with the MD simulations of Kalinichev and co-workers [19].

The 2D self-diffusion coefficient for the water in each layer was determined from the gradient of $\left\langle x^{2}+y^{2}\right\rangle$ as a function of $t$ for model MD4. Model MD4 is of $20 \mathrm{~ns}$ duration and statistical averaging is taken over MD trajectories every $0.025 \mathrm{~ns}$. The calculation of the 2D self-diffusion coefficient is performed at time $t$ for water which is located in the specific layer throughout the time period $0-t$. Thus, a spin that leaves the specified layer is excluded from the calculation even if it has returned to the layer at time $t$.

The water molecules contained in L1 are bonded to the surface of the crystal and to the aqueous $\mathrm{Ca}^{2+}$ ions. Thus the calcium ions located on the surface increase the surface affinity of the water contained in L1, as predicted in Ref. [19]. Water molecules in L1 remained immobile within the $20 \mathrm{~ns}$ simulation time.

The water molecules in L2 form the first hydration shell of adsorbed $\mathrm{Ca}^{2+}$ ions. The density of water-oxygen atoms in $\mathrm{L} 2$ and the aqueous $\mathrm{Ca}^{2+}$ ions are presented in Fig. 5, which clearly identifies the regions occupied by the water. Density also exists in circular regions which arises due to the cooperative movement of groups of water molecules mediated by the calcium. A more detailed analysis of this unusual diffusion mechanism will be published elsewhere. The

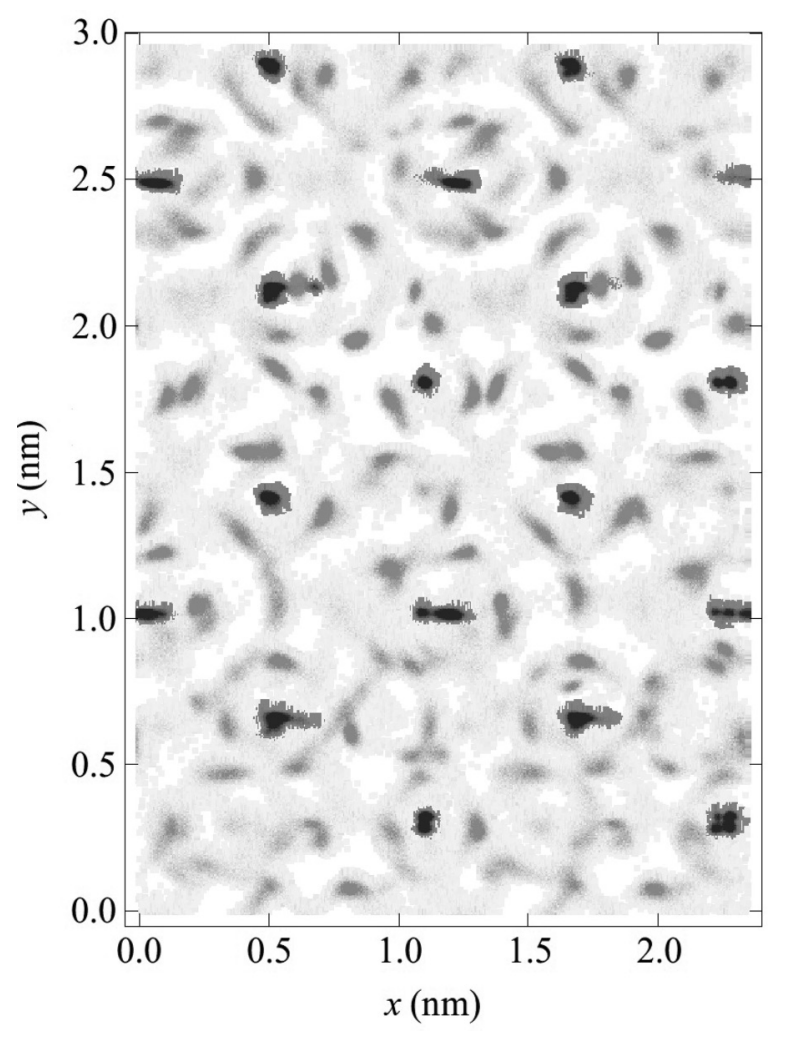

FIG. 5. The probability density of the water-oxygen atoms (lightgray shading) and calcium ions (dark-gray shading) in layer L2 for model MD4. 
diffusion coefficient for the water in layer L2 is found to be approximately $2 \times 10^{-12} \mathrm{~m}^{2} / \mathrm{s}$. The corresponding diffusion correlation time $\tau_{\mathrm{L} 2}$ defined as $\delta^{2} / 4 D$, where $\delta=0.27 \mathrm{~nm}$, is $9 \mathrm{~ns}$, which is broadly consistent with values of $\tau_{m}$ from fits to experimental data using the SL model $[4,5]$. We speculate that it is the water in L2 rather than L1 which dominates the dipolar relaxation in cementitious systems.

The water contained in the TL is fast moving, the particle probability density is uniform, and the diffusion coefficient of $1.4 \times 10^{-9} \mathrm{~m}^{2} / \mathrm{s}$ is of the same order as bulk water. The corresponding diffusion correlation time $\tau_{\mathrm{TL}}$ is $13 \mathrm{ps}$. Finally, the diffusion coefficient for the bulk water in region $\mathrm{B}$ is found to be $2.7 \times 10^{-9} \mathrm{~m}^{2} / \mathrm{s}$, which is larger than the experimental diffusion coefficient of bulk water at room temperature, $2.2 \times$ $10^{-9} \mathrm{~m}^{2} / \mathrm{s}$ [42], but consistent with MD simulations of bulk water using the same interatomic potential with a similar cell size $[22,43]$. The corresponding diffusion correlation time is about 7 ps.

The results for the surface residency time $\tau_{s}$ are presented in Table I for all models. For all models MD1-MD5, $\tau_{s}$ was determined by counting the fraction of spins remaining in the region $\mathrm{L} 1+\mathrm{L} 2$ as a function of time and executing a fit to $A e^{-t / \tau_{a}}+B e^{-t / \tau_{b}}$. Spins leaving region $\mathrm{L} 1+\mathrm{L} 2$ were excluded from the remaining total even if they subsequently returned to $\mathrm{L} 1+\mathrm{L} 2$. The double exponential fit is chosen to accommodate different desorption rates from L1 and L2. $\tau_{s}$ is selected as the longest of $\tau_{a}$ and $\tau_{b}$.

The surface residency time is found to be $0.03 \mathrm{~ns}$ or less in models MD1-MD3, indicating that the water molecules in L1+L2 desorb quite rapidly if the surface affinity is low. $\tau_{s}$ is found be $12 \mathrm{~ns}$ for model MD4 and $0.08 \mathrm{~ns}$ for MD5. The difference between the CLAYFF-SPC/E and Freeman-TIP4P potential sets of MD4 and MD5, respectively, is surprisingly large. The Freeman-TIP4P combination is normally used for clays and provided an unphysical flattened crystal interface, which was only partially corrected by modifications proposed by Galmarini [35]. We conclude that the CLAYFF-SPC/E potential set is the best combination for hydrated silica-based surface studies and note that this is the same potential set used for the MD simulations of Kalinichev and co-workers [19]. Finally, we note that there is no evidence of large-scale surface diffusion prior to desorption in any model.
Notwithstanding the spread of values from the MD simulations and a significant uncertainty in the surface morphology of C-S-H pore surfaces, all values of the surface residency time $\tau_{s}$ are approximately $2-5$ orders of magnitude less than typical values of order 1-10 $\mu$ s obtained by fitting the SL model to NMR relaxation measurements. The MD simulations do not therefore support the SL model in this regard.

\section{A TWO-LAYER MODEL AND REEVALUATION OF EXPERIMENTAL RESULTS}

Barberon and co-workers [5] measured $T_{1}$ for a hydrated mortar at various times after hydration to about $10.5 \mathrm{~h}$. This is a rare example of the full frequency dependence of $T_{1}^{-1}$ for a cement system to be found in the literature where the data is not renormalized to a master curve. These authors identified a frequency-independent relaxation rate for frequencies less than $22 \mathrm{kHz}$ due to the rigid lattice limit of the $I$ spins within the solid hydrates. From $10 \mathrm{~h}$ after hydration, a resonance feature appears at 3-5 MHz which was associated with water molecules bound to surface $\mathrm{Fe}^{3+}$ impurities. Between the two, Barberon and co-workers identified a bilogarithmic dependence of $T_{1}^{-1}$ on frequency. They divided the pore water into two environments, i.e., surface water and bulk water. The frequency-independent relaxation rate associated with the bulk water was estimated from the high-frequency limit of $T_{1}$ to be $55 \mathrm{~s}^{-1}$ and the contribution to the frequency-dependent $T_{1}^{-1}$ relaxation rate due to surface water was fitted using the SL model described in Sec. II B. Consequently, over the frequency range $0.02-2 \mathrm{MHz}$, the observed relaxation rate is given by

$$
T_{1, \mathrm{obs}}^{-1}=x T_{1, \mathrm{SL}}^{-1}+(1-x) T_{1, \mathrm{~B}}^{-1} \approx x T_{1, \mathrm{SL}}^{-1}+T_{1, \mathrm{~B}}^{-1},
$$

where $x$ is the fraction of spins on the surface and $T_{1, \mathrm{~B}}^{-1}=55 \mathrm{~s}^{-1}$ is the relaxation rate associated with the bulk water.

In executing the fits to the $T_{1}^{-1}$ relaxation rate, Barberon and co-workers estimated the values of certain physical quantities as listed in Table II leaving three fit parameters: $\tau_{s}, \tau_{m}$, and $x$. These authors assumed that $\tau_{s} \gg \tau_{m}$, which simplifies Eq. (6) so that it becomes independent of $\tau_{s}$. At the longest hydration time of $10.5 \mathrm{~h}$, the fit yielded $\tau_{m} \approx 1.3 \mathrm{~ns}$ and $x \approx 0.1$. Later, McDonald and co-workers [6] undertook a $T_{1}-T_{2}$ correlation study of hydrated cement paste. The ratio $T_{2} / T_{1}$ was found

TABLE II. List of parameters required to fit the SL model to NMR relaxation measurements on hydrated mortar [5] and cement paste [8].

\begin{tabular}{|c|c|c|}
\hline Parameter & Best estimate & Comments \\
\hline$\delta$ & $0.27 \mathrm{~nm}$ & $\begin{array}{l}\text { Estimated distance of nearest approach of a water proton } \\
\text { to a substitutional surface } \mathrm{Fe}^{3+} \text { impurity. }\end{array}$ \\
\hline$\sigma_{s}$ & $1.8 \times 10^{12} \mathrm{Fe}^{3+} / \mathrm{cm}^{2}$ & $\begin{array}{l}\text { Mass density of paramagnetic impurities measured by ESR. } \\
\text { Surface density } \sigma_{s} \text { assumes uniform density and average } \\
\text { distance between layers of } \mathrm{Fe}^{3+} \text { of } 0.6 \mathrm{~nm} \text { [5]. }\end{array}$ \\
\hline$\epsilon$ & $0.38 \mathrm{~nm}$ & Size of water molecule. \\
\hline$\chi \epsilon$ & $1 \mathrm{~nm}$ & $\begin{array}{l}\text { Thickness of surface water layer estimated from } \\
\text { calorimetry and NMR studies [47]. }\end{array}$ \\
\hline$R_{1 b}$ & $55 \mathrm{~s}^{-1}$ & Estimated contribution to the relaxation rate due to bulk water [5]. \\
\hline$x \approx N_{S} / N$ & $\approx 0.1$ & A fit parameter [5]. \\
\hline$\tau_{m}$ & $1.3 \mathrm{~ns}$ & A fit parameter assuming $\tau_{s} \gg \tau_{m}[5]$ \\
\hline$\tau_{s}$ & $13 \pm 5 \mu \mathrm{s}$ & $\begin{array}{l}\text { A fit parameter. Value from } T_{1}-T_{2} \text { NMR correlation experiments } \\
\text { on cement paste }[6,8] \text {. }\end{array}$ \\
\hline
\end{tabular}




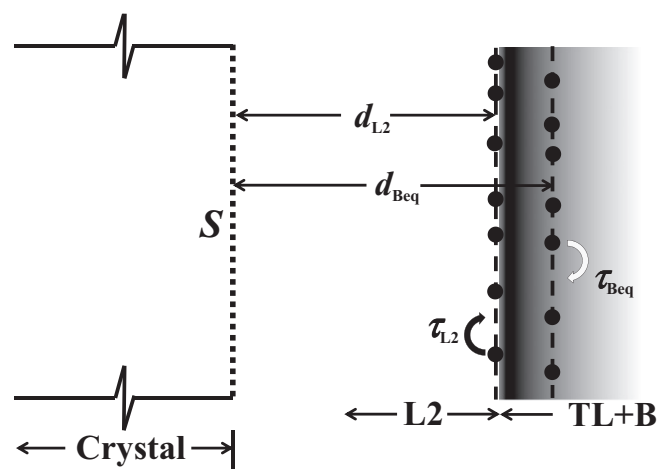

FIG. 6. A schematic representation of the two-layer (2L) model. Water diffuses with a correlation time $\tau_{\mathrm{L} 2}$ in layer L2 and $\tau_{\mathrm{Beq}}$ in an equivalent layer which is representative of the layers TL+B combined. The shading illustrates the increasing contribution to the dipolar correlation function $G(t)$ of spins in the bulk due to the interaction with surface paramagnetic impurities.

to be approximately 0.25 and the SL model was fitted to data yielding $\tau_{s} \approx 13 \mu \mathrm{s}$.

We now present the alternative model, the two-layer (2L) model, represented schematically in Fig. 6. The underlying principles which led to the SL model are retained in the $2 \mathrm{~L}$ model, that is, the primary relaxation mechanism is associated with the translational diffusion of water protons moving in a 2D plane parallel to a crystal surface containing a dilute concentration of surface paramagnetic impurities.

The $2 \mathrm{~L}$ model contains three key differences from the SL model. First, the 2L model incorporates two 2D layers of diffusing spins, i.e., one associated with slow-moving spins close to the pore surface and the second associated with fast-moving spins in the bulk of the pore. While this can likely be viewed quite generally, it is particularly appropriate to cement C-S-H where pores are believed to be substantially planar. Second, whereas the single 2D layer in the SL model contains both the diffusing spins and the $\mathrm{Fe}^{3+}$ ions, the $2 \mathrm{~L}$ model displaces the two 2D layers of diffusing spins from the layer containing the paramagnetic impurities. This is an important distinction because $G(t)$ at long times, as described in Sec. II C, is of the form $t^{-2}$ rather than $t^{-1}$. The third key difference between the SL model and the $2 \mathrm{~L}$ model relates to assumptions about the behavior of spins which depart a layer. In the SL model, spins are assumed to desorb from the surface layer with a characteristic desorption time $\tau_{s}$. The desorbed spins cease to contribute to the relaxation thereafter, which means that the SL model assumes that spins do not return to the surface layer over the time scale of a NMR relaxation time. Given relaxation times of the order of $100 \mu$ s and diffusivities of the order of $10^{-9} \mathrm{~m}^{2} / \mathrm{s}$, the implication therefore is that pore sizes must be of the order of $\mu \mathrm{m}$. Such a large size is at odds with independent and NMR observations that suggest gel pores are nanosized $[13,14]$. The $2 \mathrm{~L}$ model, by contrast, allows spins to depart the layer to return at a later time.

The $2 \mathrm{~L}$ model is developed as follows. Consider two ensembles of spins: slow-moving spins located close to the pore surface and fast-moving spins located in the bulk of the pore. The observed relaxation rate is given by Eq. (5) in the limit of fast exchange. The $2 \mathrm{~L}$ model identifies the slow-moving spins with layer L2 and the fast-moving spins with regions $\mathrm{TL}+\mathrm{B}$. Therefore, the observed relaxation rate $T_{1, \mathrm{obs}}^{-1}$ is given by

$$
T_{1, \mathrm{obs}}^{-1}=\frac{N_{\mathrm{L} 2}}{N} T_{1, \mathrm{~L} 2}^{-1}+\frac{N_{\text {bulk }}}{N} T_{1, \text { bulk }}^{-1},
$$

where $N \approx N_{\mathrm{L} 2}+N_{\text {bulk }}$ is the total number of particles in the pore and $N_{\text {bulk }} \approx N$ refers to the combined number of spins contained in the TL and B layers. There is a similar expression for $T_{2 \text { obs }}^{-1}$. Spins in layer L1 are temporarily neglected.

The rate $T_{1 \text {,bulk }}^{-1}$ contains contributions due to the $I-I$ and $I-S$ interactions with the relative contributions dependent on the size of the pore. For pores sufficiently large such that, over the NMR time scale, bulk water molecules do not sample the full volume of the pore, the measured relaxation rate will be dominated by $T_{1 \text {,bulk, } I I}^{-1}$. Here it is assumed that pore sizes are sufficiently small such that the relaxation due to the $I-S$ interaction dominates the $I-I$ contribution in both the bulk and in L2. Therefore, Eq. (18) may be written as

$T_{1, \mathrm{obs}}^{-1}=x T_{1, \mathrm{~L} 2, I S}^{-1}+(1-x) T_{1, \mathrm{bulk}, I S}^{-1} \approx x T_{1, \mathrm{~L} 2, I S}^{-1}+T_{1, \mathrm{bulk}, I S}^{-1}$,

where $x=N_{\mathrm{L} 2} / N$ and, in principle, provides an estimate of the pore size. We use the results presented in Sec. IIC to provide an estimate for $T_{1, \mathrm{~L} 2, I S}^{-1}$ and $T_{1, \text { bulk, } I S}^{-1}$.

The $2 \mathrm{~L}$ model is illustrated schematically in Fig. 6. Some model parameters are estimated from the MD simulation results and some quantities taken from Barberon and coworkers [5], as summarized in Table III. $T_{1, \mathrm{~L} 2, I S}^{-1}$ is associated with the slow-moving spins in L2. The dipolar correlation function is calculated using Eq. (14) and is dependent upon the choice of $\tau_{\mathrm{L} 2}$ and $d_{\mathrm{L} 2}=\epsilon \xi_{\mathrm{L} 2}$, where we take $\epsilon=\delta=0.27$ $\mathrm{nm}$ as the distance of closest approach of two proton spins. $d_{\mathrm{L} 2}$ is chosen to be $2 \delta$ guided by the MD simulation results presented in Fig. 4 which show a sharp peak in the spin density

TABLE III. List of parameters required to fit the 2L model to NMR relaxation measurements on hydrated mortar [5] and cement paste [8].

\begin{tabular}{lcl}
\hline \hline Parameter & Best estimate & \\
\hline$\delta$ & $0.27 \mathrm{~nm}$ & Comments \\
$\sigma_{s}$ & $1.8 \times 10^{12} \mathrm{Fe}^{3+} / \mathrm{cm}^{2}$ & Approximate distance of nearest approach of two protons in water. \\
$d_{\mathrm{L} 2}$ & $2 \delta$ & From Barberon et al. [5]. \\
$d_{\mathrm{Beq}}$ & $2.21 \delta$ & From MD simulation, this work. \\
$x$ & & A falculation, this work. \\
$\tau_{\mathrm{L} 2}$ & & A fit parameter, see Eq. (19). \\
$\tau_{\mathrm{Beq}}$ & A fit parameter. \\
\hline \hline
\end{tabular}


due to the mobile spins in L2 at a distance approximately $2 \delta$ from the crystal surface. $\tau_{\mathrm{L} 2}$ is a fit parameter.

The second 2D layer is designed to approximate $T_{1, \text { bulk, } I S}^{-1}$ for the combined effect of the fast-moving spins contained in both the TL and $\mathrm{B}$ regions. The reduction of the quasitwo-dimensional (Q2D) volume of spins to a representative equivalent 2D layer is a significant simplification forced, in part, by the absence of a full Q2D solution for the dipolar correlation function. However, we justify the simplification by noting, first, that $G_{I S}(t) \propto t^{-2}$ at long times for both the Q2D layer and 2D equivalent layer and, second, that $G_{I S}(0) \propto z^{-4}$ which means that the spins that provide the greatest contribution to the relaxation are those in a layer closest to the crystal surface.

The second layer is characterized by $d_{\text {Beq }}$ and $\tau_{\text {Beq }}$, where the subscript refers to a bulk equivalent layer. An estimate for $d_{\text {Beq }}$ can be obtained by noting that $G(0) \propto z^{-4}$. If we make the assumption that the areal density of spins $\sigma_{\text {Beq }}$ in the equivalent layer is the same as that in the bulk, such that $\sigma_{\text {Beq }}=\delta N_{v}$ where $N_{v}$ is the bulk volume density of $I$ spins, it is possible to determine $d_{\text {Beq }}$ by solving

$$
\frac{\delta N_{v}}{d_{\mathrm{Beq}}^{4}}=N_{v} \int_{2 \delta}^{\infty} z^{-4} d z,
$$

from which $d_{\text {Beq }}=2.21 \delta$. In other words, the $2 \mathrm{D}$ equivalent layer is placed at a position $z=2.21 \delta$ where the contribution to $G(0)$ is the same as the average contribution to $G(0)$ of the spins in the $\mathrm{TL}+\mathrm{B}$ region of the pore, assuming that the areal spin density of water in the layer is characteristic of bulk water. It is emphasized that the $2 \mathrm{~L}$ model allows all spins to contribute to the relaxation at all times. The contribution is characterized by $d_{\mathrm{L} 2}$ and $\tau_{\mathrm{L} 2}$, or by $d_{\mathrm{Beq}}$ and $\tau_{\mathrm{Beq}}$, depending on where the spin is located at any one time.

The $2 \mathrm{~L}$ model is fitted to $T_{1}^{-1}$ as a function of frequency over the range 0.02-2 $\mathrm{MHz}$ measured by Barberon and coworkers [5] for a hydrated mortar for the longest hydration time of $10.5 \mathrm{~h}$. The $2 \mathrm{~L}$ model requires three fit parameters similar to the SL model for a complete fit to the frequency dependence of $T_{1}^{-1}$ and the ratio $T_{2} / T_{1}$. In this case, the three parameters are $\tau_{\mathrm{L} 2}, \tau_{\mathrm{Beq}}$, and $x$, as summarized in Table III. To execute the fit, values of $\tau_{\mathrm{L} 2}$ and $\tau_{\mathrm{Beq}}$ are chosen and the best fit is obtained by varying the single parameter $x$. The quality of fit (QoF) is obtained from an unweighted leastsquares calculation. Figure 7 presents the QoF as a function of $\tau_{\mathrm{L} 2}$ and $\tau_{\mathrm{Beq}}$ over five orders of magnitude for each diffusion correlation time. The black shading indicates a QoF in the range 138-145 (arbitrary units) and the dark gray shading corresponds to a QoF in the range 146-180. The upper QoF limit of 180 was chosen as the approximate value obtained by fitting the SL model to the same data. Other combinations of correlation times $\tau_{\mathrm{L} 2}$ and $\tau_{\mathrm{Beq}}$ either produced poorer fits or it was not possible to produce a fit at all. A good fit to the data of Barberon and co-workers is obtained using the $2 \mathrm{~L}$ model provided the two diffusion correlation times $\tau_{\mathrm{L} 2}$ and $\tau_{\mathrm{Beq}}$ differ by about three orders of magnitude. The optimum fits, that is, QoF $\leqslant 145$, are obtained for $\tau_{\mathrm{L} 2}$ in the range 40-130 ns and $\tau_{\mathrm{Beq}}$ in the range $25-45 \mathrm{ps}$.

The ratio $T_{2} / T_{1}$ has been found to lie in the range $0.25-0.30$ for a range of cementitious and other materials

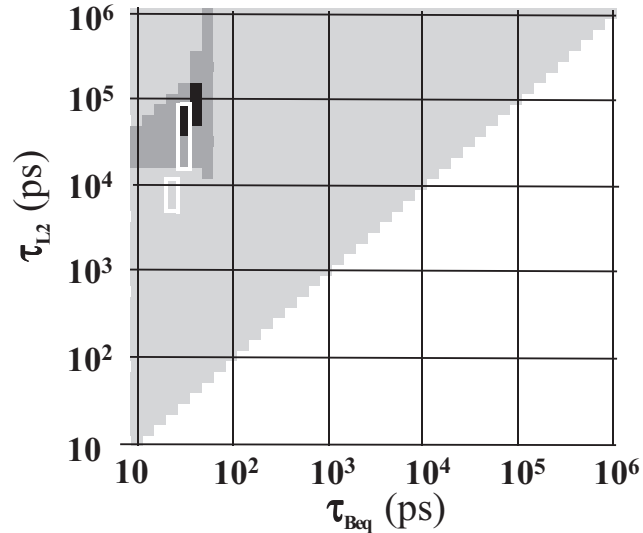

FIG. 7. The quality-of-fit (QoF) parameter (see text) is plotted as a function of $\tau_{\mathrm{L} 2}$ and $\tau_{\mathrm{Beq}}$. The black squares indicate QoF $<$ 145 , the dark-gray squares indicate QoF in the range 146-180, and the light-gray squares indicate $\mathrm{QoF}>180$. The white lines contain results which simultaneously have a $\mathrm{QoF}<180$ and a ratio $T_{2} / T_{2}$ in the range $0.25-0.30$.

$[6,44]$. Values of $T_{2} / T_{1}$ in the range $0.25-0.30$ are indicated by the white boundary in Fig. 7 and overlaps with the best fits to the experimental data, providing confidence in the $2 \mathrm{~L}$ model. $T_{2} / T_{1}$ has a weak dependence on $\tau_{\mathrm{L} 2}$ but a strong dependence on $\tau_{\text {Beq. }}$. A similar observation was made by McDonald and co-workers [6] in applying the SL model with regard to $\tau_{m}$ and $\tau_{s}$, respectively. The dual requirements of a QoF $\leqslant 180$ and $T_{2} / T_{1}$ in the range $0.25-0.30$ are satisfied by $\tau_{\mathrm{L} 2}=18-75$ ns and $\tau_{\text {Beq }}=28-40 \mathrm{ps}$. Both results are strikingly consistent with the results of simulation MD4 presented in Sec. III which yielded diffusion correlation times of $9 \mathrm{~ns}$ and $13 \mathrm{ps}$ for layers L2 and TL, respectively.

The SL model fitted to the same data of Barberon et al. [5] yields a QoF of 181 over the same frequency range using $\tau_{m}=1.3 \mathrm{~ns}$. A comparison of the two models is presented in Fig. 8. We make the observation that the SL model also provides a fit that is a weak function of $\tau_{m}$. Although Barberon et al. quoted $1.3 \mathrm{~ns}$ as the surface diffusion correlation time, a value of $\tau_{m}$ in the range $0.5-1.3 \mathrm{~ns}$ also provides a fit of similar quality over the frequency range $0.02-2 \mathrm{MHz}$.

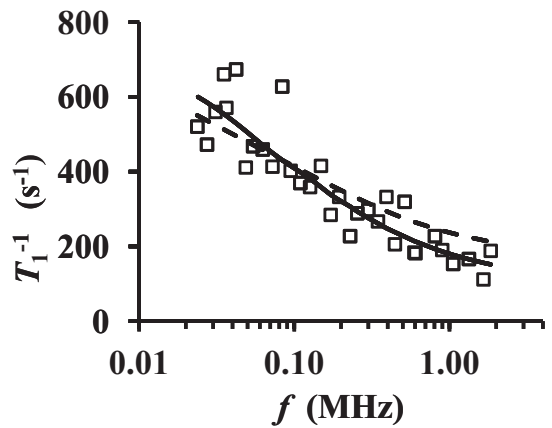

FIG. 8. $T^{-1}$ is plotted as a function of NMR frequency. The experimental data $(\square)$ are taken from Barberon and co-workers [5] for a hydrated mortar. The fit obtained using the SL model (dashed line) and 2L model (solid line) are shown. 
It is tempting to associate the fit parameter $x$ in Eq. (19) with the fraction of spins located in the surface region following Eq. (5) thereby allowing an estimate of typical pore size. The combined requirement of $\mathrm{QoF}<180$ and $T_{2} / T_{1}$ in the range $0.25-0.30$ produces $x$ in the range $0.0015-0.0047$, suggesting typical pore sizes of $100 \mathrm{~nm}$ or more. Barberon and co-workers obtain a value of about $20 \mathrm{~nm}$ which is closer to measured estimates $[13,45]$. However, Barberon and co-workers set $\epsilon=0.38 \mathrm{~nm}$ [5] and if we assume $\epsilon=0.38 \mathrm{~nm}$ for the $2 \mathrm{~L}$ model, the revised fits also yield an estimate of $20 \mathrm{~nm} . \epsilon$ is a distance unit which scales $G(t)$ and, where possible, is chosen to allow the model $G(0)$ to match the value of $G(0)$ obtained from another source, for example, from MD [22]. Models for $G(t)$, including the SL and 2L models, assume a uniform spin density which is a poor approximation at short distances where the $I-I$ pair correlation function is highly structured. $\epsilon$ may therefore be chosen to compensate for the shortcoming of the uniform spin density approximation. If so, one would expect $\epsilon \lesssim \delta$. Notwithstanding, Barberon and co-workers set $\epsilon$ approximately equal to the "water molecule size" of $0.38 \mathrm{~nm}$ [5]. The physical basis for this choice is not explained.

However, we question the validity of estimating the pore size from $x$ for other reasons. For example, there exists a significant uncertainty in the value of the density of paramagnetic impurities, $\sigma_{S}$. This is estimated from electron spin resonance (ESR) measurements and the surface density is deduced from the dry mass of sample. It is assumed that the surface density of impurities is the same as in the bulk. There is evidence, however, of clustering of iron in AFm and AFt phases [46] which means that the actual value of $\sigma_{S}$ may be smaller. Moreover, although the average spin density of layer L2 is similar to bulk water, the MD simulations suggest that not all of the water molecules located in L2 are equally mobile, indicating that just a fraction of the water in L2 contributes significantly to the relaxation. Both effects would decrease the pore-size estimate obtained from $x$.

Finally, we reiterate that the SL model requires a surface residency time $\tau_{s}$, whereas the $2 \mathrm{~L}$ model does not. In the SL model, the spins are assumed to desorb from the surface and cease to contribute to the relaxation thereafter, with the fraction of spins remaining on the surface decaying as $\exp \left(-t / \tau_{s}\right)$. A motivation for the present study was that $\tau_{s}$ obtained using the SL model is unacceptably long. The $2 \mathrm{~L}$ model, however, does not require the introduction of the $\exp \left(-t / \tau_{s}\right)$ term because $G(t)$ at long times is of the form $t^{-2}$, which can be Fourier transformed, whereas $G(t)$ decays as $t^{-1}$ in the SL model (which cannot be Fourier transformed). The $2 \mathrm{~L}$ model therefore resolves the surface residency time discrepancy.

\section{CONCLUSIONS}

The results of MD simulations of hydrated Q2D pores with the water confined by a silica or tobermorite crystal are presented. The water surface residency time is found to be about 2-5 orders of magnitude shorter than values that consistently emerge when the SL models of Korb and co-workers are fitted to experimental NMR relaxation rate data [2-9]. Furthermore, no MD simulation reveals largescale surface diffusion of water prior to desorption. Both observations conflict with the SL model.
The MD simulations identify three highly structured layers for water at the surface of a tobermorite pore when the bridging tetrahedra are replaced by $\mathrm{Ca}^{2+}$ ions. Layer $\mathrm{L} 1$ contains water adhered to the surface of the pore, layer L2 is the first hydration shell of the aqueous $\mathrm{Ca}^{2+}$ ions located on the L1/L2 boundary, and the third layer is a transition layer. The fourth layer is bulklike water in the central region of the pore.

Guided by the MD simulations, a two-layer (2L) model is proposed and used to interpret NMR relaxation rates obtained from frequency-dependent $T_{1}^{-1}$ data obtained by Barberon and co-workers [5] for a hydrated mortar and the ratio $T_{2} / T_{1}$ obtained from $T_{1}-T_{2}$ correlation studies of hydrated cement paste by McDonald and co-workers [6]. The 2L model retains the principles that underly the SL model, that is, the dipolar relaxation is associated with the interaction of diffusing $I$ spins moving in a 2D plane with rare static paramagnetic $S$ spins located at the surface of the confining crystal. In essence, the surface residency time $\tau_{s}$ and diffusion correlation time $\tau_{m}$ of the SL model are replaced by diffusion correlation times $\tau_{\mathrm{L} 2}$ and $\tau_{\text {Beq }}$ in the new $2 \mathrm{~L}$ model. In the $2 \mathrm{~L}$ model, the correlation times are associated with water diffusing in two 2D planes representative of regions $\mathrm{L} 2$ and the TL+bulk, respectively.

The theory is presented for the dipolar correlation function $G(t)$ for a 2D layer of diffusing spins displaced from paramagnetic relaxation centers located in the surface of the confining crystal. It is shown that the long-time behavior of $G(t)$ is proportional to $t^{-2}$, in contrast to the $t^{-1}$ behavior when the relaxation centers are contained in the same plane as the diffusing spins, which forms the basis of the SL model.

Using the $2 \mathrm{~L}$ model, a satisfactory fit to the frequencydependent $T_{1}^{-1}$ data obtained by Barberon and co-workers [5] for a hydrated mortar can only be obtained if $\tau_{\mathrm{L} 2}$ and $\tau_{\mathrm{Beq}}$ are of the order of nanoseconds and picoseconds, respectively. The fits are significantly better than those obtained from the SL model using the same experimental data. Using this experimental data only, the best fits $(\mathrm{QoF} \leqslant 145)$ are obtained for $\tau_{\mathrm{L} 2}$ and $\tau_{\mathrm{Beq}}$ in the range $40-130 \mathrm{~ns}$ and $25-45 \mathrm{ps}$, respectively. Remarkably, the values of $\tau_{\mathrm{L} 2}$ and $\tau_{\mathrm{Beq}}$ that satisfy the stringent requirement that the ratio $T_{2} / T_{1}$ lies in the range $0.25-0.30$ coincides with the best fits to the frequency-dependent $T_{1}^{-1}$ data. This provides confidence in the validity of the model. The combined requirements and accepting fits such that $\mathrm{QoF} \leqslant 180$ suggest that $\tau_{\mathrm{L} 2}=18-75 \mathrm{~ns}$ and $\tau_{\mathrm{Beq}}=28-40 \mathrm{ps}$.

The $2 \mathrm{~L}$ model yields NMR relaxation times which not only provide an improved fit to the experimental data compared to the SL model but yield values of $\tau_{\mathrm{L} 2}$ and $\tau_{\mathrm{Beq}}$ broadly consistent with simulation. Model MD4 produces values of $9 \mathrm{~ns}$ and $13 \mathrm{ps,}$ respectively. Considering the uncertainties in the morphology of $\mathrm{C}-\mathrm{S}-\mathrm{H}$, the aqueous $\mathrm{Ca}^{2+}$ density, the crudeness of the interatomic potentials, the difficulty of obtaining accurate diffusion coefficients even in well-characterized systems, and other poorly known physical quantities, the agreement can be considered as good.

In conclusion, the MD simulations provide fresh insight into the dynamics of water at silicate-based surfaces and the 2L model provides a different approach to the interpretation of NMR relaxation dispersions obtained from proton-bearing porous silicate-based systems such as porous glasses, zeolites, cementitious materials, and oil-bearing rocks. 


\section{ACKNOWLEDGMENTS}

N.C.H. and J.S.B. acknowledge the support of Engineering and Physical Sciences Research Council (UK) for finan- cial support (Grant No. EP/H033343/1). S-H.P.C. acknowledges support from the European Union Seventh Framework Programme (FP7/2007-2013) under Grant Agreement No. 264448 .
[1] N. Bloembergen, E. M. Purcell, and R. V. Pound, Phys. Rev. 73, 679 (1948).

[2] J.-P. Korb, S. Xu, and J. Jonas, J. Chem. Phys. 98, 2411 (1993).

[3] J.-P. Korb, M. Whaley-Hodges, and R. G. Bryant, Phys. Rev. E 56, 1934 (1997).

[4] S. Godefroy, J.-P. Korb, M. Fleury, and R. G. Bryant, Phys. Rev. E 64, 021605 (2001).

[5] F. Barberon, J.-P. Korb, D. Petit, V. Morin, and E. Bermejo, Phys. Rev. Lett. 90, 116103 (2003).

[6] P. J. McDonald, J.-P. Korb, J. Mitchell, and L. Monteilhet, Phys. Rev. E 72, 011409 (2005).

[7] A. Plassais, M.-P. Pomiés, N. Lequeux, J.-P. Korb, D. Petit, F. Barberon, and B. Bresson, Phys. Rev. E 72, 041401 (2005).

[8] L. Monteilhet, J.-P. Korb, J. Mitchell, and P. J. McDonald, Phys. Rev. E 74, 061404 (2006).

[9] J.-P. Korb, New J. Phys. 13, 035016 (2011).

[10] K. Brownstein and C. Tarr, J. Magn. Reson. 26, 17 (1977).

[11] A. J. Allen, J. J. Thomas, and H. M. Jennings, Nat. Mater. 6, 311 (2007).

[12] E. Masoero, E. D. Gado, R. J.-M. Pellenq, S. Yip, and F.-J. Ulm, Soft Matter 10, 491 (2014).

[13] H. M. Jennings, Cem. Conc. Res. 38, 275 (2008).

[14] A. Valori, P. J. McDonald, and K. L. Scrivener, Cem. Conc. Res. 49, 65 (2013).

[15] I. G. Richardson, Cem. Conc. Res. 34, 1733 (2004).

[16] R. Kirkpatrick, A. Kalinichev, X. Hou, and L. Struble, Mater. Struc. 38, 449 (2005).

[17] R. J.-M. Pellenq, A. Kushima, R. Shahsavari, K. J. Van Vliet, M. J. Buehler, S. Yip, and F.-J. Ulm, Proc. Natl. Acad. Sci. 106, 16102 (2009).

[18] G. Kovačević, B. Persson, L. Nicoleau, A. Nonat, and V. Veryazov, Cem. Conc. Res. 67, 197 (2015).

[19] A. G. Kalinichev, J. Wang, and R. J. Kirkpatrick, Cem. Conc. Res. 37, 337 (2007).

[20] J.-P. Korb, P. McDonald, L. Monteilhet, A. Kalinichev, and R. Kirkpatrick, Cem. Conc. Res. 37, 348 (2007).

[21] A. Abragam, The Principles of Nuclear Magnetism (Oxford University Press, Oxford, 1961).

[22] D. A. Faux, P. J. McDonald, N. C. Howlett, J. S. Bhatt, and S. V. Churakov, Phys. Rev. E 87, 062309 (2013).

[23] I. S. Gradshteyn and I. M. Ryzhik, Table of Integrals, Series, and Products, 7th ed., edited by Alan Jeffrey and Daniel Zwillinger (Elsevier/Academic, Amsterdam, 2007).
[24] S. Merlino, E. Bonaccorsi, and T. Armbruster, Eur. J. Mineral. 13, 577 (2001).

[25] S. V. Churakov, Am. Mineral. 94, 156 (2009).

[26] S. V. Churakov, Eur. J. Mineral. 21, 261 (2009).

[27] W. Smith and T. Forester, J. Molec. Graphics 14, 136 (1996).

[28] I. T. Todorov, W. Smith, K. Trachenko, and M. T. Dove, J. Mater. Chem. 16, 1911 (2006).

[29] H. J. C. Berendsen, J. R. Grigera, and T. P. Straatsma, J. Phys. Chem. 91, 6269 (1987).

[30] J. Abascal and C. Vega, J. Chem. Phys. 123, 234505 (2005).

[31] R. T. Cygan, J.-J. Liang, and A. G. Kalinichev, J. Phys. Chem. B 108, 1255 (2004).

[32] N. H. de Leeuw, G. W. Watson, and S. C. Parker, J. Chem. Phys. 99, 17219 (1995).

[33] N. H. de Leeuw, S. C. Parker, C. R. A. Catlow, and G. D. Price, Phys. Chem. Minerals 27, 332 (2000).

[34] C. L. Freeman, J. H. Harding, D. J. Cooke, J. A. Elliott, J. S. Lardge, and D. M. Duffy, J. Phys. Chem. C 111, 11943 (2007).

[35] S. Galmarini, Ph.D. thesis, Ecole Polytechnique Federale de Lausanne, 2014.

[36] M. P. Allen and D. J. Tildesley, Computer Simulation of Liquids (Oxford University Press, Oxford, 1989).

[37] J. S. Bhatt, P. J. McDonald, D. A. Faux, N. C. Howlett, and S. V. Churakov, Int. J. Quant. Chem. 114, 1220 (2014).

[38] P. Ewald, Ann. Phys. 369, 253 (1921).

[39] W. G. Hoover, Phys. Rev. A 31, 1695 (1985).

[40] S. V. Churakov, C. Labbez, L. Pegado, and M. Sulpizi, J. Phys. Chem. C 118, 11752 (2014).

[41] L. Cheng, P. Fenter, K. L. Nagy, M. L. Schlegel, and N. C. Sturchio, Phys. Rev. Lett. 87, 156103 (2001).

[42] K. Krynicki, C. D. Green, and D. W. Salter, Faraday Discuss. Chem. Soc. 66, 199 (1978).

[43] S. Kerisit and C. Liu, Env. Sci. Technol. 43, 777 (2009).

[44] S. Haber-Pohlmeier, S. Stapf, D. V. Dusschoten, and A. Pohlmeier, Open Magn. Reson. J. 3, 57 (2010).

[45] A. C. A. Muller, K. L. Scrivener, A. M. Gajewicz, and P. J. McDonald, J. Phys. Chem. C 117, 403 (2013).

[46] H. F. W. Taylor, Cement Chemistry (Telford, London, 1997).

[47] J. J. Fripiat, M. Letellier, and P. Levitz, Philos. Trans. R. Soc. London A 311, 287 (1984). 